\title{
THE STUDY OF OBSERVER VARIATION IN THE RADIOLOGICAL CLASSIFICATION OF PNEUMOCONIOSIS
}

\author{
BY
}

\author{
J. W. J. FAY and J. R. ASHFORD
}

From the Scientific Department, National Coal Board

(RECEIVED FOR PUBLICATION NOVEMBER 26, 1959)

In a long-term investigation such as the National Coal Board's Pneumoconiosis Field Research (P.F.R.), it is essential to establish satisfactory and stable procedures for making the necessary observations and measurements. It is equally important regularly to apply suitable methods of checking the accuracy and consistency of the various observations and measurements. One aspect of vital importance in the P.F.R. is the classification of the series of chest radiographs taken, at intervals, of all the men under observation. This is inevitably a subjective process, and (as with other similar fields of work) it is desirable to obtain some understanding of the basic process behind the operation. This can usefully be done by the help of "models" designed to describe the process, if necessary in simplified terms. The problem of the radiological classification of pneumoconiosis has been studied hitherto in terms of coefficients of disagreement (interobserver variation) and inconsistency (intra-observer variation), but for various reasons the method was not considered entirely satisfactory. New methods of approach were therefore developed for studying the performance of the two doctors responsible for the film reading in the Research, and two distinct "models" were derived. The advantages and disadvantages of each are described in the paper, together with the applications of the two models to the study of some of the problems arising in the course of the investigation.

The first model is based on the assumption that if a film is selected at random from a batch representing a whole colliery population, and that if the film is of "true" category $i$, the chance of its being read as another category $(j)$ is a constant, $p_{i j}$, which depends upon the observer concerned, the particular batch of films being read, and the values of $i$ and $j$. This model enables the performance of the readers to be monitored satisfactorily, and it has also been used to investigate different methods for arriving at an agreed, or "definitive", assessment of radiological abnormality. The $p_{i j}$ model suffers from the disadvantage of applying only to "average" films, and the assumptions made are such that it manifestly does not provide an entirely realistic representation of the reading process on any particular film.

The second "improved" model was therefore developed to overcome this criticism. Briefly, it is considered that each film is representative of a unique degree of abnormality, located on a continuum, or abnormality scale, which covers the whole range of simple pneumoconiosis. The scale of abnormality is then chosen in such a way that, whatever the true degree of abnormality of the film, the observer's readings will be normally distributed about the true value with constant bias and variability at all points along the scale. The very large number of readings available has been analysed to determine the optimum positions of the category boundaries on the abnormality scale and in this way the scale has been unambiguously defined. The model enables the routine reading standards to be monitored, and it has also been used to investigate the underlying distribution of abnormality at individual collieries. Its chief disadvantage is the extensive computational work required.

The "fit" of both models to the data collected in the Research is shown to be satisfactory and on balance it appears that both have applications in this field of study. The method chosen in any given circumstance will depend upon the particular requirement and the facilities available for computational work. 
Two of the objectives of the National Coal Board's Pneumoconiosis Field Research (Fay, 1957) are (1) to express the present prevalence of pneumoconiosis at about 25 selected collieries in terms of its various stages of severity as measured by radiological examination, and (2) to investigate the progression of pneumoconiosis at these collieries, also in terms of radiological examination. It was therefore essential, at the beginning of the Research, to agree upon uniform procedures to be adopted by the two medical officers responsible for taking and reading the radiographs.

A good deal of experience and advice was available, particularly from members of the Pneumoconiosis Research Unit (P.R.U.) of the Medical Research Council, and after careful consideration it was decided that each film should be read independently at least once by each doctor. When these routine readings agreed the common assessment was taken as the "definitive" classification. In the event of disagreement the film was read by both doctors in consultation and the "definitive" classification was agreed upon by joint discussion. This procedure is such that the "easy" films are disposed of quickly but the more "difficult" ones are classified only after joint discussion by the two readers.

It is generally accepted that the classification of radiographs for pneumoconiosis is subject to variation (Fletcher and Oldham, 1949), and it is therefore necessary to maintain a check upon the performance of the two doctors, as regards both agreement with one another and consistency within their own performances from time to time. The available methods for analysing the data were not considered to be entirely satisfactory and early in the Research a relatively simple mathematical model was developed as an aid to the assessment of the readings (Ashford, 1956). Although useful for many purposes this model applies only to the study of "average" effects, as represented by randomly selected single films or by large numbers of films, and not to particular individual films. To obtain a more exact representation of the reading process a more complicated model, based on the concept of a continuous scale of abnormality, was therefore developed (Ashford, 1959).

This paper describes the application of the two models to a number of problems concerned with the reading of radiographs which have arisen during the course of the Pneumoconiosis Field Research. The methods cover only two out of a number of possible ways of representing the film reading process in quantitative terms and alternative procedures are currently under consideration both by the National Coal Board and by the Pneumoconiosis Research Unit.

\section{Method of Approach}

Reading radiographs for pneumoconiosis is a complex procedure, involving the recognition and interpretation of the characteristic opacities on a background of other lung markings, and a precise mathematical description of the process would be impracticable. As in many similar fields of study it is, however, helpful to consider the problem in terms of a simple theoretical model (or models) to represent the process of classification in mathematical terms. On the basis of the theoretical model it is then possible to derive a quantitative measure of the accuracy of the reading process.

Any acceptable model must satisfy three main requirements:-

(a) It must "fit" the observed data. That is to say, the results obtained by assuming that the model is true must agree with the actual observations.

(b) The general assumptions involved in the model must be consistent with the nature of the reading process, i.e. the model must be realistic if it is to assist those concerned with making the observations.

(c) It must be relatively simple, both in conception and application, as far as this is possible.

The choice of one particular model rather than another must represent a compromise between these three considerations. Although requirements (b) and (c) are to some extent a matter of opinion, an objective measure may be obtained of requirement (a). Thus, other things being equal, the most acceptable model is the one which provides the best "fit" to the observations.

\section{Classification of Films}

The categories of pneumoconiosis used in the radiological surveys are based on the P.R.U. classification, and are those which were currently accepted by international agreement, which recognizes a distinction between "simple" and "complicated" pneumoconiosis (International Labour Office, 1953).*

Simple pneumoconiosis is divided on a quantitative basis into three categories, increasing in degree of abnormality and numbered 1 to 3 , category 0 being reserved for films that are within normal limits. The lower limit of the continuum of abnormality is represented by a film of a person who has never been exposed to airborne dust, showing none of the specific changes associated with simple pneumoconiosis, and the upper limit by a film on which no further increase of simple pneumoconiosis can be detected. The location of the various

* The International Labour Office, 1953, classification has since been revised, but the revised criteria were not used for the work described in this paper. 
categories on this continuum is subjective. The boundaries between categories 1 and 2 and between categories 2 and 3 are defined by means of standard films which illustrate borderline cases. Category 1 is defined by means of standard films which represent typical examples of the category (Fletcher and Oldham, 1951).

Complicated pneumoconiosis (Progressive Massive Fibrosis or P.M.F.) is divided separately into four categories-A, B, C and D. P.M.F. is commonly believed to occur only on a background of at least category 2 of simple pneumoconiosis and it differs qualitatively from the latter condition. In no sense can any of the categories of P.M.F. be said to lie on the continuum of simple pneumoconiosis, although it might be asserted that a film showing evidence of P.M.F. must have reached at least the lower boundary of category 2 .

When the surveys were first started each film was read three times, twice by the doctor responsible for the survey at the particular colliery (each was responsible for the surveys at half the collieries, divided roughly on a regional basis (Fay, 1957)) and once by the other doctor. This procedure was later modified in the light of experience (see below) and the second reading by the doctor responsible for the survey was discontinued.

\section{Previous Method of Analysis}

At the beginning of the research use was made of such experience as had been reported in the literature (Fletcher and Oldham, 1949) and the analysis of the film readings was based on two main concepts, those of "agreement" (with the definitive category) and "consistency" (between repeat readings by the same observer), which were expressed in quantitative terms as coefficients of disagreement and inconsistency respectively. The calculation of these coefficients was based on the assumption that the categories of pneumoconiosis may be treated as real numbers, which further implies that the categories are linearly related. That is to say, the difference between one category and the next is the same whatever the categories concerned, and hence the differences are additive. In view of the nature of the process of radiograph classification the validity of this assumption, especially with reference to the relationship between complicated pneumoconiosis and the various categories of simple pneumoconiosis, is subject to doubt. Statistical tests of the significance of the difference between corresponding coefficients were based on the extension of methods of analysis for continuous distributions to the essentially discrete distributions of film readings. The validity of this extension is also subject to some doubt on mathematical grounds. For these reasons the available methods of analysis were considered to be far from satisfactory, and alternative procedures were investigated.

\section{The " $p_{i j}$ " Model}

The " $p_{i j}$ " model (Ashford, 1956) is based on the assumption that there exists a unique "true" category associated with any given film, this true category being defined as the opinion which would be obtained most frequently in a long series of joint readings by the two observers in consultation. The model then assumes that, if a film is selected at random from a batch representing a complete colliery population, and if the film belongs to true category $i$, the chance of reading the film as category $j$ is a constant, $p_{i j}$, where $p_{i j}$ depends on the observer concerned, the particular batch of films being read, and the values of $i$ and $j$. For example, if an observer is examining a film which happens to belong to true category 0 , it is assumed that the chance of his reading it as category 0 is a constant $\left(p_{o o}\right)$, depending on the performance of the observer at the time of reading and on the batch of films concerned. If the observer is reading accurately, $p_{0 o}$ will be close to unity, whereas $p_{01}$ (the chance of the true category 0 film being read as category 1 ) will be correspondingly small, and $p_{0_{2}}, p_{0_{3}}, p_{0_{4}}$, and $\mathrm{p}_{05}$ will be negligible.

For the purposes of the model, category A of P.M.F. is considered as a separate category (labelled "4") and categories B, C, and D are taken together as a single category (labelled "5"). Thus the model is applied to both simple and complicated pneumoconiosis. In that no difference is made between two distinct aspects of the reading process it is obvious that some measure of validity has been sacrificed in the interests of simplicity of treatment.

As regards the classification of simple pneumoconiosis, it is evident that the chance of reading any single film as one category rather than another is highest when the film is near a category boundary. In the extreme case of a film which is exactly on the boundary between two categories it seems reasonable to suppose that the observer will be equally likely to assign it to the higher or lower category in the absence of reading bias. Indeed, this model obviously represents a simplification of the actual process of classification and in any particular case it may be expected that the probability of error will vary according to whether or not the film is on the borderline between two categories. Again, the model applies only to studies of the variations associated with the readings on films representing complete colliery populations (with which the 
TABLE 1

TEST OF THE "p $p_{i j}$ " MODEL WITH THE OBSERVED DATA NUMBERS OF OCCURRENCES (OBSERVED AND EXPECTED) OF THE VARIOUS SEQUENCES OF ROUTINE READINGS (All films)

\begin{tabular}{|c|c|c|c|c|c|c|c|c|c|c|}
\hline Colliery & \multicolumn{2}{|c|}{$C_{2}$} & \multicolumn{2}{|c|}{ C. } & \multicolumn{2}{|c|}{ C. } & \multicolumn{2}{|c|}{$\mathrm{C}_{3}$} & \multicolumn{2}{|c|}{$C_{11}$} \\
\hline $\begin{array}{l}\text { Prevalence of } \\
\text { Category } 1 \text { or More }\end{array}$ & \multicolumn{2}{|c|}{$42 \%$} & \multicolumn{2}{|c|}{$33 \%$} & \multicolumn{2}{|c|}{$21 \%$} & \multicolumn{2}{|c|}{$9 \%$} & \multicolumn{2}{|c|}{$5 \%$} \\
\hline Sequence & Observed & Expected & Observed & Expected & Observed & Expected & Observed & Expected & Observed & Expected \\
\hline $\begin{array}{l}(0,0,0) \\
(0,0,1) \\
(0,1,0) \\
(1,0,0) \\
(0,1,1) \\
(1,0,1) \\
(1,1,0) \\
(1,1,1) \\
(1,1,2) \\
(1,2,1) \\
(1,2,2) \\
(2,1,2) \\
(2,2,1) \\
(2,2,2) \\
(3,3,3) \\
(4,4,4) \\
(5,5,5)\end{array}$ & $\begin{array}{r}361 \\
21 \\
43 \\
18 \\
19 \\
4 \\
36 \\
28 \\
16 \\
11 \\
5 \\
11 \\
8 \\
13 \\
21 \\
13 \\
15 \\
29\end{array}$ & $\begin{array}{c}365 \\
18 \\
46 \\
17 \\
12^{*} \\
2 \\
28 \\
46^{*} \\
14 \\
9 \\
4 \\
10 \\
7 \\
8 * \\
25 \\
13 \\
16 \\
29\end{array}$ & $\begin{array}{r}436 \\
5 \\
37 \\
28 \\
4 \\
2 \\
43 \\
18 \\
4 \\
12 \\
3 \\
9 \\
2 \\
21 \\
30 \\
8 \\
16 \\
5\end{array}$ & $\begin{array}{c}426 \\
1 * \\
40 \\
34 \\
6 \\
4 \\
31^{*} \\
19 \\
2 \\
11 \\
7 \\
12 \\
4 \\
13 * \\
22 \\
6 \\
16 \\
4\end{array}$ & $\begin{array}{r}1,337 \\
46 \\
12 \\
16 \\
13 \\
9 \\
32 \\
54 \\
23 \\
7 \\
0 \\
10 \\
2 \\
18 \\
33 \\
11 \\
18 \\
14\end{array}$ & $\begin{array}{c}1,341 \\
31^{*} \\
13 \\
15 \\
21^{*} \\
16^{*} \\
27 \\
50 \\
11^{*} \\
8 \\
5^{*} \\
16 \\
6 \\
10^{*} \\
27 \\
9 \\
12 \\
14\end{array}$ & $\begin{array}{r}1,336 \\
56 \\
15 \\
13 \\
11 \\
6 \\
36 \\
27 \\
11 \\
3 \\
1 \\
2 \\
2 \\
4 \\
10 \\
2 \\
1 \\
1\end{array}$ & $\begin{array}{c}1,313 \\
49 \\
38 * \\
36 \\
11 \\
10 \\
13^{*} \\
27 \\
7 \\
4 \\
2 \\
3 \\
2 \\
3 \\
8 \\
2 \\
1 \\
1\end{array}$ & $\begin{array}{r}1,167 \\
24 \\
5 \\
9 \\
3 \\
5 \\
5 \\
13 \\
7 \\
0 \\
0 \\
0 \\
1 \\
4 \\
8 \\
3 \\
3 \\
2\end{array}$ & $\begin{array}{r}1,165 \\
23 \\
7 \\
12 \\
5 \\
6 \\
6 \\
3 \\
9 \\
3^{*} \\
2 \\
2 \\
3 \\
3 \\
3 \\
1 * \\
6 \\
2 \\
2 \\
2\end{array}$ \\
\hline All others & 87 & 90 & 44 & 69* & 70 & 93* & 29 & 36 & 10 & 13 \\
\hline
\end{tabular}

* Value of $x^{2}$ in excess of 3.0.

Pneumoconiosis Field Research is mainly concerned). Readings on specially selected batches of films may well follow a different pattern. This means that the model is intended to refer only to the study of "average" effects based on large numbers of films representing complete colliery populations and should not be extended to cover any particular film.

If the true distribution of abnormality in a batch were known, estimates of the probabilities, $p_{i j}$, would be given by the proportion of films of true category $i$ which were read as category $j$. In practice, however, the true category of any particular film cannot be obtained and the estimates of $p_{i j}$ must be calculated in terms of the proportion of films of definitive category $i$ which are read as category $j$ on the particular set of readings. Although it is shown subsequently that the definitive category may be incorrect on an appreciable proportion of films, the overall distribution of abnormality in terms of the definitive readings is reasonably close to the true situation as defined above, and hence the use of the definitive readings to represent the true values is justified in the application of the model.

In the light of these considerations the question arises as to how well the model fits the facts. This may best be examined by using the observed values of $p_{i j}$ to compare the actual numbers of occurrences of the various sequences of the three routine readings $[$ e.g. $(1,0,2)$, denoting category 1 on the first reading by the observer responsible for the survey, 0 on his second reading and 2 on the reading by the other observer] with the corresponding numbers which would be expected if the model were exact.* Details are given in Table 1, which shows the results obtained at five typical collieries, covering a wide range of prevalence of pneumoconiosis as found at the selected collieries. In view of the effect of random fluctuations the data corresponding to sequences for which the expected number of occurrences is very small have been pooled under the heading "All other sequences". The results indicate that the model does provide a reasonable "fit" to the observed data. There is a tendency for the observed numbers of films assigned to the sequences $(0,0,1),(1,1,0),(1,1,2)$ and $(2,2,1)$ to be slightly higher than the expected numbers. These sequences have in common the feature that the duplicate readings by the doctor responsible for the survey are consistent and differ from the single reading by the other doctor. The calculation of the expected numbers of films assigned to each of the sequences is based on the assumption that the three routine readings are independent. The tendency for the duplicate readings to be consistent points to the fact that there is a slight association between the first and second readings by the doctor who carries out the survey and that, in these circumstances, the hypothesis of independence is not strictly true.

* The probability of reading a film of true category $i$ as $(j, k, 1)$ is

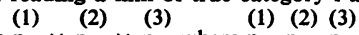

given by the expression $p_{i j} \times p_{1 k} \times p_{i 1}$, where $p_{i j}, p_{1 k}, p_{11}$ refer to the reading probabilities on the first, second and third sets of routine readings. 
Possibly the doctors tend to "remember" their previous readings on a small proportion of the "doubtful" films, although the second set of duplicate readings is generally carried out several months after the first. On balance, however, it is considered that this slight "memory effect" does not detract from the validity of the model, particularly in view of the fact that it is now the practice for only one routine reading to be made by the doctor responsible for the survey.

Viewed in the light of the requirements outlined earlier it is clear that the main criterion for an acceptable model is satisfied in that the results obtained by its application fit the facts reasonably well. With regard to the general assumptions involved in the model it is apparent that it does not provide an entirely realistic representation of the reading process on any particular film. This is a limitation, but in the circumstances it is impossible for a simple model accurately to represent the distinct processes involved in reading both simple and complicated pneumoconiosis. The third requirement, i.e. simplicity of conception and application, is also satisfied reasonably well. The calculations involved in the estimation of the reading probabilities are straightforward, although the use of a set of 36 values of $p_{i j}$ to represent the performance by an observer on a particular batch of films is rather clumsy.

\section{Applications of the " $p_{\mathrm{ij}}$ " Model}

In spite of its limitations, the $\mathrm{p}_{\mathrm{ij}}$ model is a useful concept and has a number of applications in a study such as the Pneumoconiosis Field Research. Some of these applications are illustrated below.

Comparison of Reading Standards. - The $p_{i j}$ values corresponding to any particular set of readings provide a convenient summary of the observer's performance. A typical example is given in Table 2, which shows the $p_{i j}$ values recorded on the three sets of routine readings (one by Observer $A$ and two by Observer B) on the films obtained at one colliery. On this particular occasion there were considerable differences in the reading levels of the two doctors. The values of $p_{00}$ show that the probability of Observer A reading a category 0 film correctly was about 0.93, compared with the corresponding figures of 0.98 and 0.99 for Observer B's first and second readings. The probability of reading a category 0 film as category 1 was 0.07 on Observer A's reading and 0.02 and 0.01 on Observer B's first and second readings respectively. Comparison of other values of $p_{i j}$ shows that Observer $A$ tended, on this occasion, to read higher than Observer B throughout the whole range of simple pneumoconiosis. On the other hand, Observer B's first and second readings are in close agreement.

When dealing merely with different sets of readings on the same batch of films the tables of $p_{i j}$ values are effectively equivalent to the more familiar "two-way" tables (Ashford, 1960), by which any two sets of readings may be compared. Even here, however, the use of probabilities rather than numbers of films is distinctly more helpful in the interpretation of the results.

When the $p_{i j}$ values corresponding to readings on different batches of films are compared it is found that the differences from colliery to colliery are smaller than the differences between different sets of readings at the same colliery. In general, the variations do not appear to be directly related to variations in the distribution of abnormality, apart from a slight tendency for the values of $p_{00}$ to increase with increasing proportions of normal films. In this connexion it should be borne in mind that the $p_{i j}$ values are a reflection of the variation of the readings on films of the same category. When the variation of the readings on all the films within a given batch is considered there is a definite tendency for the consistency to fall as the proportion of abnormal films increases (Ashford, 1960).

The Accuracy of the Individual Readings.-As the differences in the values of $p_{i j}$ between the various collieries are relatively small it is considered that the results may profitably be combined for the purpose of any general examination of the film reading process. Details of the average $p_{i j}$ values obtained

TABLE 2

"p ${ }_{i j}$ " VALUES CORRESPONDING TO THE ROUTINE READINGS AT A COLLIERY

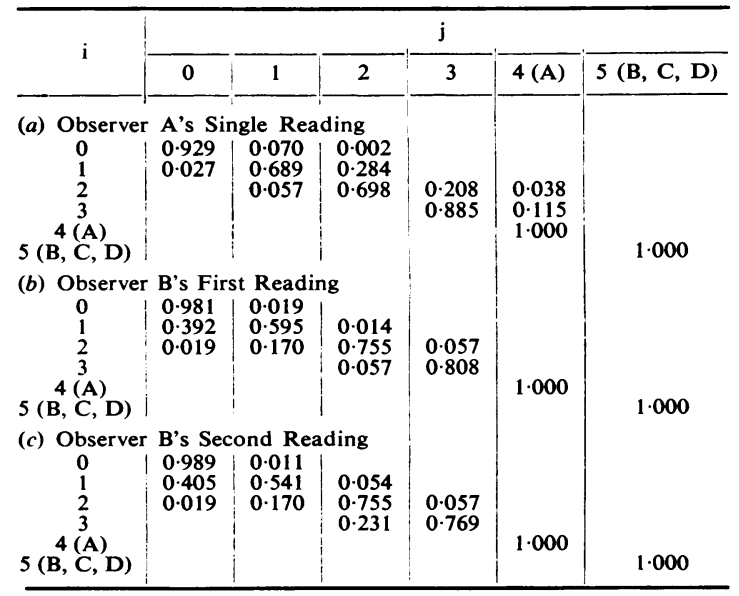


on the initial surveys at all the selected collieries are given in Table 3. These results are based on the analysis of about 100,000 film readings by the two doctors, and the $p_{i j}$ values given refer to the average performance of the readers on films selected at random from a typical colliery population.

The highest standard of performance is obtained on films of category 0 , on which $97 \%$ of the single readings are in agreement with the definitive classification. The corresponding figures for categories $1,2,3,4(\mathrm{~A})$ and $5(\mathrm{~B}, \mathrm{C}, \mathrm{D})$ are respectively $63 \%, 69 \%, 79 \%, 77 \%$ and $90 \%$. The majority of errors are of one category only, although there is an appreciable chance that films of definitive category A may be assigned to any of the other categories on a single reading.

It will be noted from Table 3 that there is a consistent tendency for the values (of $p_{i j}$ ) lying below the diagonal to be larger than the corresponding values (of $\mathrm{p}_{\mathrm{ji}}$ ) lying above the diagonal. For example, the values of $p_{10}$ and $p_{01}$ are respectively 0.250 and 0.029 . This means that there is a chance of 250 in 1000 (or 1 in 4) that a film of true category 1 will be read as category 0 compared with a chance of only 29 in 1000 that a film of true category 0 will be read as category 1 . This trend is a consequence of the fact that in the batches of films considered the numbers of films tend to decrease with increasing radiological category (Fig. 1). Thus, the majority of the "borderline" films in any given category lie on the lower boundary of the category and there is therefore a greater chance of assigning the film to a lower category rather than to a higher.

Investigation of Alternative Procedures for Assessing the Definitive Classification.-During the first three years of the Research the definitive classification was obtained on the basis of three sets of routine readings, two by the doctor responsible for the survey and one by the other doctor. A considerable amount of film reading was therefore involved, and as soon as a sufficiently reliable body of information about the errors associated with the film reading process had been built up it was decided to in-

TABLE 3

" $p_{1 j}$ " VALUES (BASED ON ROUTINE READINGS ON FIRST ROUND OF MEDICAL SURVEYS)

\begin{tabular}{c|c|c|c|c|c|c}
\hline & \multicolumn{5}{|c|}{$\mathrm{j}$} \\
\cline { 2 - 6 } & 0 & 1 & 2 & 3 & 4 (A) & 5 (B, C, D) \\
\hline 0 & 0.970 & 0.029 & 0.001 & & & \\
1 & 0.250 & 0.629 & 0.110 & 0.001 & 0.009 & 0.001 \\
2 & 0.023 & 0.176 & 0.691 & 0.078 & 0.030 & 0.001 \\
3 & 0.001 & 0.005 & 0.173 & 0.789 & 0.032 & 0.001 \\
4 (A) & 0.027 & 0.050 & 0.068 & 0.031 & 0.768 & 0.056 \\
5 (B, C, D) & 0.006 & 0.003 & 0.004 & & 0.092 & 0.895 \\
\hline
\end{tabular}

vestigate the relative accuracy of the various alternative procedures. The following five possible methods of obtaining the definitive classification were examined.

Method A.-Each film to be read once only by the Unit Medical Officer responsible for the survey and the "definitive" category to be assigned on the basis of this single reading. Under this procedure the need for classification by joint discussion would not arise.

METHOD B.-Each film to be read once by each of the Unit Medical Officers. If the two routine readings are consistent the common reading to be taken as "definitive". In any case of disagreement the film to be classified by joint discussion.

Merhod C.-Each film to be read three times, twice by the Unit Medical Officer responsible for the survey and once by the other Unit Medical Officer. If the three routine readings are consistent the common reading to be taken as "definitive". In any case of disagreement the film to be classified by joint discussion.

This corresponds to the procedure at that time in operation.

MethoD D.-Each film to be read three times, twice by the Unit Medical Officer responsible for the survey and once by the other Unit Medical Officer. If the three routine readings do not differ by more than one category the majority reading to be taken as "definitive". In any case of disagreement of more than one category the "definitive" classification to be assigned by joint discussion.

METHOD E.-Each film to be read three times, twice by the Unit Medical Officer responsible for the survey and once by the other Unit Medical Officer. If the three routine readings are all simple pneumoconiosis, the category nearest to the average reading to be taken as "definitive". If the three routine readings are all the same category of complicated pneumoconiosis the common reading to be taken as "definitive". If the readings include both simple and complicated pneumoconiosis, or more than one category of complicated pneumoconiosis, the film to be classified by joint discussion.

By the use of the $p_{i j}$ model it is possible directly to determine the errors associated with the definitive category when this is derived straight from the routine readings. However, classification by joint discussion is involved in all methods except $A$, and it is therefore necessary to have some knowledge of the errors associated with joint classification. On the basis of the results obtained from a series of special reading sessions undertaken by the doctors it was estimated that about $80 \%$ of the readings by joint discussion (on the films selected for this method of classification) are correct (Ashford, 1957). This result has therefore been assumed for the purpose of comparing the methods. Details of the calculations 


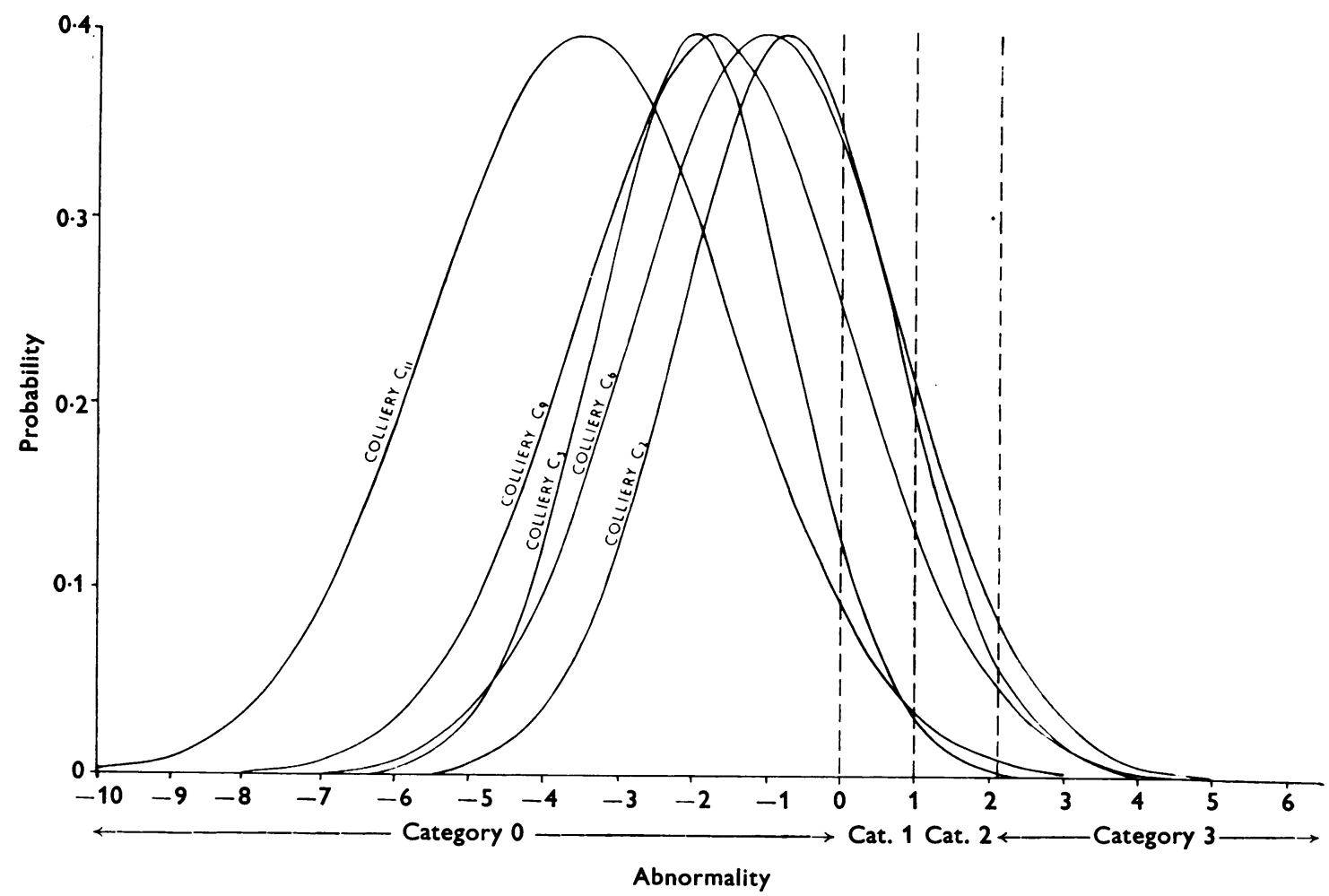

FIG. 1-Underlying distributions of abnormality.

involved are given in Appendix I and the proportion of films in the various categories which would be incorrectly classified on the definitive readings is given in Table 4. Method A involves a relatively high proportion of misclassifications but there is little to choose between methods B, C, D, and E. Method $B$ involves the same number of readings by each doctor, as compared with methods C, D, and $\mathrm{E}$, which involve two readings by one doctor and one by the other. Hence the introduction of a bias due to differences in standards between the readers is eliminated with method B, but not with the other three methods. For this reason it was decided to adopt method B on future surveys, in spite of the fact that it involves a slight loss of accuracy in comparison with some of the other procedures.

\section{The "Improved" Model}

Although the $\mathrm{p}_{\mathrm{ij}}$ model was considered to provide a sufficiently good approximation to enable several urgent problems to be tackled in the early days of the Research, its limitations were recognized and further work was carried out in an endeavour to obtain a more exact representation of the reading process. In view of the fundamental differences in the criteria of diagnosis for simple and complicated pneumoconiosis it was felt that the next logical step was to consider each separately. Attention was therefore concentrated on the reading of simple pneumoconiosis only, on the grounds that the great majority of films belong to this category, and the improved model is concerned solely with this aspect of the problem.

Each radiograph may be regarded as being representative of a certain unique degree of ab-

TABLE 4

CLASSIFICATION OF RADIOGRAPHS A COMPARISON OF FIVE DIFFERENT PROCEDURES PERCENTAGE OF FILMS ASSIGNED TO AN INCORRECT "DEFINITIVE" CATEGORY

\begin{tabular}{c|c|c|c|c|c|c|c}
\hline \multirow{2}{*}{ Method } & $\begin{array}{c}\text { No. of } \\
\text { Routine } \\
\text { Readings }\end{array}$ & \multicolumn{1}{|c|}{0} & \multicolumn{5}{|c|}{ "True" Category } \\
\cline { 3 - 8 } & & 1 & 2 & 3 & A & B.C.D. \\
\hline A & 1 & 4 & 37 & 32 & 20 & 23 & 13 \\
B & 2 & 1 & 18 & 14 & 9 & 9 & 6 \\
C & 3 & 2 & 16 & 14 & 10 & 11 & 7 \\
D & 3 & $<1$ & 18 & 13 & 8 & 9 & 5 \\
E & 3 & $<1$ & 16 & 17 & 11 & 11 & 7 \\
\hline
\end{tabular}


normality, which may be denoted the "true" abnormality of the film. For any given film this true abnormality value must be located at some point on a continuum, or abnormality scale, which covers the whole range of simple pneumoconiosis. It is perhaps unfortunate that the categories of simple pneumoconiosis have been labelled $0,1,2$ and 3 , as these numbers have no arithmetical significance in this context. The use of the numerical labels for the categories has led to the growth of a "tradition" by which it has become customary to regard category 0 as covering the range between 0 and 1 on the "natural" abnormality scale, category 1 as covering the range between 1 and 2 , and category 2 as covering the range between 2 and 3 . In this spirit it might seem logical to regard category 3 as lying between 3 and 4 on the scale, but some authorities (Wise, 1957) suggest that category 3 should extend upwards without limit from the poirt 3 on the abnormality scale. An abnormality scale of this kind is not an invalid one, but it has none of the desirable properties with which a more logically constructed scale of measurement is endowed.

When choosing a scale of abnormality to cover the subjective process of radiograph reading it is perhaps helpful to consider the design of an instrument for making an objective measurement. In a situation of this kind the scale of measurement is usually pre-defined (for example, a speedometer may be expected to give a reading in miles per hour). If it is required to make the measurement over a certain range, the instrument should be designed so that the readings in this range may all be made with equal accuracy, and so that, if there is any systematic bias, this will be constant at all points throughout the range. In the case of the speedometer the degree of "needle wobble" and "drift" should be the same at all speeds. In reading radiographs the situation is reversed. The "instrument" (represented by the reader) is pre-defined, in the sense that there is little chance of making a fundamental change in the performance of an experienced reader (provided, of course, no deliberate attempt is made to do so) but the scale of measurement may be varied at will. However, the basic requirement is the same, in that the bias and variability of the readings by any particular observer should remain constant at all points along the scale of measurement. Unfortunately, there is no reason why the "traditional" scale described above should have these properties.

Thus, the first requirement for an improved procedure is to choose the scale of abnormality in such a way that, whatever the true abnormality value of a film, the observer's readings will be distributed about this value with constant bias and variability.
In mathematical terms this means that if the true abnormality value of the film is $\mathrm{x}$, the readings by an observer on the film will be distributed with mean $x+b$ and variance $\sigma^{2}$ where $b$ and $\sigma^{2}$ represent respectively his reading bias and variability and are constant at all points of the scale. Now, the process of film reading involves the consideration and interpretation of a large number of different aspects of the film, and on these grounds it seems reasonable to require that the error distribution should be normal (Gaussian), as is commonly found in the case of objective measurements of all kinds made under similar circumstances. In other words, the scale of abnormality should be chosen in such a way that the readings on any particular film are normally distributed about the true abnormality value of the film, with constant bias and variability at all points along the scale.

The data available for consideration consist of the three sets of routine readings and the definitive readings at each colliery. These sets of readings may be regarded as resulting from the superposition of the error distribution associated with the performance of the observer, at the time the readings were made, upon the "true" distribution of abnormality at the colliery concerned. Examination of the definitive and routine readings obtained at the selected collieries shows that it is possible to choose the position of the boundaries between the categories in such a way that the observed distributions are approximately normal. This means that for each set of readings the result of superimposing the error distribution on the true distribution is a normal distribution. It was therefore decided to test as a first step the hypothesis that the true distributions are also normal. Experience has shown that many characteristics of the mining populations under consideration follow a normal distribution and the assumption that the true distributions of radiological abnormality at the various collieries are also normal is not an unreasonable one. On this hypothesis the true distribution of abnormality at a colliery can be described in terms of two parameters, the mean $\mathrm{m}$ and the variance $\mathrm{s}^{2}$, and the distribution of observations on any particular set of routine readings (which represents a normal error distribution superimposed on a true normal distribution) has mean $(m+b)$ and variance $\left(\sigma^{2}+s^{2}\right)$.

For practical purposes, the readings are made in terms of four discrete categories which together cover the whole of the range of abnormality, and the choice of a scale of measurement resolves itself into the selection of suitable positions for the category boundaries. As the lowest category of simple pneumoconiosis is category 0 and the highest is category 3 it is reasonable to take the lower 
TABLE 5

TEST OF THE "IMPROVED" MODEL WITH THE OBSERVED DATA NUMBERS OF OCCURRENCES (OBSERVED AND EXPECTED) OF THE VARIOUS SEQUENCES OF ROUTINE READINGS (Simple Pneumoconiosis Only)

\begin{tabular}{|c|c|c|c|c|c|c|c|c|c|c|}
\hline \multirow{2}{*}{$\begin{array}{l}\text { Colliery } \\
\text { Sequence }\end{array}$} & \multicolumn{2}{|c|}{$\mathrm{C}_{2}$} & \multicolumn{2}{|c|}{$\mathrm{C}_{6}$} & \multicolumn{2}{|c|}{ C, } & \multicolumn{2}{|c|}{$\mathrm{C}_{3}$} & \multicolumn{2}{|c|}{$\mathrm{C}_{11}$} \\
\hline & Observed & Expected & Observed & Expected & Observed & Expected & Observed & Expected & Observed & Expected \\
\hline $\begin{array}{l}(0,0,0) \\
(0,0,1) \\
(0,1,0) \\
(1,0,0) \\
(0,1,1) \\
(1,0,1) \\
(1,1,0) \\
(1,1,1) \\
(1,1,2) \\
(1,2,1) \\
(2,1,1) \\
(1,2,2) \\
(2,1,2) \\
(2,2,1) \\
(2,2,2) \\
(3,3,3)\end{array}$ & $\begin{array}{r}361 \\
21 \\
43 \\
18 \\
19 \\
4 \\
36 \\
28 \\
16 \\
11 \\
5 \\
11 \\
8 \\
13 \\
21 \\
13\end{array}$ & $\begin{array}{c}358 \\
23 \\
35 \\
14 \\
19 \\
8 \\
26^{*} \\
36 \\
13 \\
16 \\
7 \\
12 \\
5 \\
14 \\
23 \\
15\end{array}$ & $\begin{array}{r}436 \\
5 \\
37 \\
28 \\
4 \\
2 \\
43 \\
18 \\
4 \\
12 \\
3 \\
9 \\
2 \\
21 \\
30 \\
8\end{array}$ & $\begin{array}{c}468 \\
3 \\
33 \\
22 \\
7 \\
3 \\
30^{*} \\
20 \\
2 \\
13 \\
8^{*} \\
4^{*} \\
2 \\
16 \\
12^{*} \\
11\end{array}$ & $\begin{array}{r}1,337 \\
46 \\
12 \\
16 \\
13 \\
9 \\
32 \\
54 \\
23 \\
7 \\
0 \\
10 \\
2 \\
18 \\
33 \\
11\end{array}$ & $\begin{array}{r}1,359 \\
45 \\
16 \\
13 \\
15 \\
9 \\
24 \\
50 \\
19 \\
7 \\
5^{*} \\
8 \\
4 \\
10^{*} \\
25 \\
14\end{array}$ & $\begin{array}{r}1,336 \\
56 \\
15 \\
13 \\
11 \\
6 \\
36 \\
27 \\
11 \\
3 \\
1 \\
2 \\
2 \\
4 \\
10 \\
2\end{array}$ & $\begin{array}{c}1,366 \\
47 \\
17 \\
16 \\
12 \\
9 \\
16^{*} \\
23 \\
9 \\
3 \\
3 \\
3 \\
2 \\
3 \\
5 * \\
1\end{array}$ & $\begin{array}{r}1,167 \\
24 \\
5 \\
9 \\
3 \\
5 \\
5 \\
13 \\
7 \\
0 \\
0 \\
0 \\
1 \\
4 \\
8 \\
3\end{array}$ & $\begin{array}{r}1,164 \\
21 \\
4 \\
7 \\
6 \\
8 \\
3 \\
13 \\
6 \\
1 \\
2 \\
2 \\
3 \\
1 * \\
6 \\
3\end{array}$ \\
\hline All others & 53 & 56 & 35 & 44 & 31 & 30 & 17 & 16 & 9 & 6 \\
\hline
\end{tabular}

* Value of $\chi^{2}$ in excess of $3 \cdot 0$.

boundary of category 0 as $-\infty$ and the upper boundary of category 3 as $+\infty$. The location and dimensions of the abnormality scale are arbitrary, in the sense that it is possible to choose any convenient zero or origin and any convenient scaling factor. This means that two of the remaining three category boundaries can be located at arbitrary points on the scale. For convenience, the boundary between categories 0 and 1 may be chosen to be zero and that between categories 1 and 2 to be unity. Only the boundary (denoted " $a$ ") between categories 2 and 3 then remains to be determined from the data. Thus the scale of abnormality is

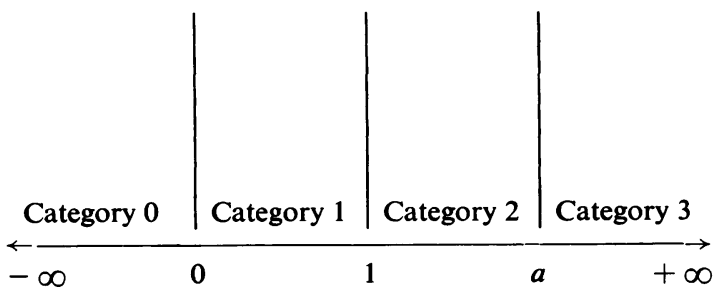

Direction of increasing abnormality $\rightarrow$

The mathematical techniques employed to determine the position of the boundary between categories 2 and 3 for the subsequent estimation of the various parameters are based on the method of maximum likelihood and have been described in detail elsewhere (Ashford, 1959). Briefly, the procedure has been, first, to derive the best estimate of $a$ from the analysis of the definitive reading distributions recorded for all the collieries. This analysis indicates that the boundary between categories 2 and 3 should be located at the point, $a=2 \cdot 12$. Next, the model so derived is used to estimate the parameters associated with the underlying abnormality distributions and with the various error distributions in terms of the number of occurrences of the different sequences of all routine readings. Finally, the validity of the model can be tested by comparing the "predicted" numbers of sequences of readings with the actual numbers obtained at individual collieries. The "fit" of the model to the data from five collieries is illustrated at Table 5. The calculations necessary for a satisfactory treatment of the data are complex and a complete solution has been obtained at only these five collieries. However, as they represent a good cross-section of the range of prevalence found at all the selected collieries, they may be taken to provide a reliable test of the general application of the procedure. It will be seen that the results obtained using the model are, in general, in close agreement with the observations. The number of the discrepancies is no greater than would be expected to occur by chance, although (as in Table 1) there is evidence of a slight "memory effect", in that the observed numbers of films assigned to the sequence on which the duplicate readings by the same doctor are consistent tend to be higher than the predicted numbers. This confirms the deduction from the earlier model that there is a slight association between the first and second readings by the doctor who carried out the survey. However, as with the earlier model, this "memory effect" does not detract from the validity of the new model for the study of observer variation. Indeed the model as enunciated applies only to a single reading on a film, and the 
observed association between duplicate readings is a secondary consideration. Comparison of the results in Table 5 with those in Table 1 shows that the general level of agreement is better with the new model than with the old.

\section{Applications of the "Improved" Model}

As with the older, $\mathrm{p}_{\mathrm{ij}}$, model, the new model has a number of useful applications in the study of radiograph reading procedures and observations, and some of these applications are illustrated below.

Underlying Distributions of Abnormality.-It has been found that, provided the scale of abnormality is suitably chosen, the data are consistent with the hypothesis that the underlying abnormality at a colliery follows a normal distribution and may therefore be defined in terms of only two parameters - the mean and standard deviation. The distributions at the five collieries considered in detail may be compared in Fig. 1, and the corresponding means and standard deviations are given in Table 6. The means have been estimated from the definitive reading distributions (as these are considered to be effectively free from bias), and the standard deviations are derived from the analyses in terms of the number of occurrences of the various sequences of routine readings. The distribution curves are then plotted by reference to tabulated values of the standard normal distribution, and a simple picture is obtained of the distribution of abnormality at the particular colliery.

TABLE 6

UNDERLYING DISTRIBUTIONS OF ABNORMALITY

\begin{tabular}{c|c|c}
\hline Colliery & Mean & Standard Deviation \\
\hline$C_{2}$ & -0.73 & 1.49 \\
$C_{8}$ & -1.05 & 1.79 \\
$C_{9}$ & -1.72 & 1.89 \\
$C_{3}$ & -1.99 & 1.33 \\
$C_{11}$ & -3.53 & 2.03 \\
\hline
\end{tabular}

The Accuracy of a Single Reading.-Under the new model it has been shown that the performance of an observer on a particular set of readings may be described in terms of two parameters, his bias and his variability. The corresponding figures for the three sets of routine readings at the five collieries are given in Table 7, where the figures for bias refer to the appropriate doctor's standard relative to the definitive readings.

The salient feature of these results is the general tendency for Observer B to read higher than Observer A. With the exception of Observer B's
TABLE 7

READING ERRORS

\begin{tabular}{|c|c|c|c|c|}
\hline \multicolumn{2}{|c|}{ Colliery (Surveyed by Observer A) } & C, & $\mathbf{C}_{\mathbf{3}}$ & $\mathrm{C}_{11}$ \\
\hline Bias (b) & $\begin{array}{l}\text { Observer A (1st reading) } \\
\text { Observer A (2nd reading) } \\
\text { Observer B }\end{array}$ & $\begin{array}{l}-0.44 \\
=0.36 \\
-0.39\end{array}$ & $\begin{array}{l}-0.08 \\
-0.03 \\
-0.07\end{array}$ & $\begin{array}{l}0.14 \\
0.08 \\
0.40\end{array}$ \\
\hline $\begin{array}{l}\text { Standard } \\
\text { error }(\sigma)\end{array}$ & $\begin{array}{l}\text { Observer A (1st reading) } \\
\text { Observer A (2nd reading) } \\
\text { Observer B }\end{array}$ & $\begin{array}{l}0.31 \\
0.21 \\
0.63\end{array}$ & $\begin{array}{l}0.37 \\
0.33 \\
0.72\end{array}$ & $\begin{array}{l}0.42 \\
0.35 \\
0.46\end{array}$ \\
\hline \multicolumn{2}{|c|}{ Colliery (Surveyed by Observer B) } & $\mathbf{C}_{\mathbf{2}}$ & \multicolumn{2}{|c|}{$\mathbf{C}_{6}$} \\
\hline$\overline{\text { Bias (b) }}$ & $\begin{array}{l}\text { Observer B (1st reading) } \\
\text { Observer B (2nd reading) } \\
\text { Observer A }\end{array}$ & $\begin{array}{l}0.13 \\
0.35 \\
0.09\end{array}$ & \multicolumn{2}{|c|}{$\begin{array}{r}-0.14 \\
0.02 \\
-0.60\end{array}$} \\
\hline $\begin{array}{l}\text { Standard } \\
\text { error }(\sigma)\end{array}$ & $\begin{array}{l}\text { Observer B (1st reading) } \\
\text { Observer B (2nd reading) } \\
\text { Observer A }\end{array}$ & $\begin{array}{l}0.41 \\
0.42 \\
0.67\end{array}$ & \multicolumn{2}{|c|}{$\begin{array}{l}0.52 \\
0.44 \\
0.47\end{array}$} \\
\hline
\end{tabular}

readings at Colliery $C_{2}$ the reported biases on the first and second readings by the doctor responsible for the survey are in close agreement. It is also apparent that the second set of readings by the doctor responsible for the survey are generally less variable than the first. Both the sets of readings by the doctor responsible for the survey tend to be less variable than the readings by the other doctor.

Besides providing a very convenient summary of standards for any given set of readings the new model enables account to be taken of the variation in the chance of misclassification within the category. This is illustrated in Fig. 2, which refers to the three sets of routine readings at Colliery $C_{11}$. For each set of readings a series of four curves shows the chance of reading a film of any given degree of abnormality as category $0,1,2$ and 3 on each of the routine readings.

As the abnormality value increases from the lower limit of category 0 the chance that Observer A's first reading is category 0 decreases from unity below about -1.3 to 0.8 at $-0.5,0.4$ at $0,0.1$ at 0.5 and approaches zero beyond about 0.9 . The chance of Observer A's calling a film category 1 on his first reading increases from zero below about $-1 \cdot 3$ to 0.6 at 0 , reaches a maximum of 0.76 at 0.4 , and then falls away to 0.4 at 1.0 , being effectively zero again beyond about $2 \cdot 0$. The category 2 curve shows a similar pattern, although the maximum of 0.82 at 1.5 is slightly higher than that for category 1 (because category 2 covers a greater range of abnormality). The curve for category 3 increases from zero below about 0.8 to 0.6 at the boundary between categories 2 and 3, and approaches unity beyond about 3.0. At any one point on the scale of abnormality the sum of the ordinates of the four curves is unity, as the film must be assigned to some category. Thus the majority of reading errors are of one category only, although in a small number 


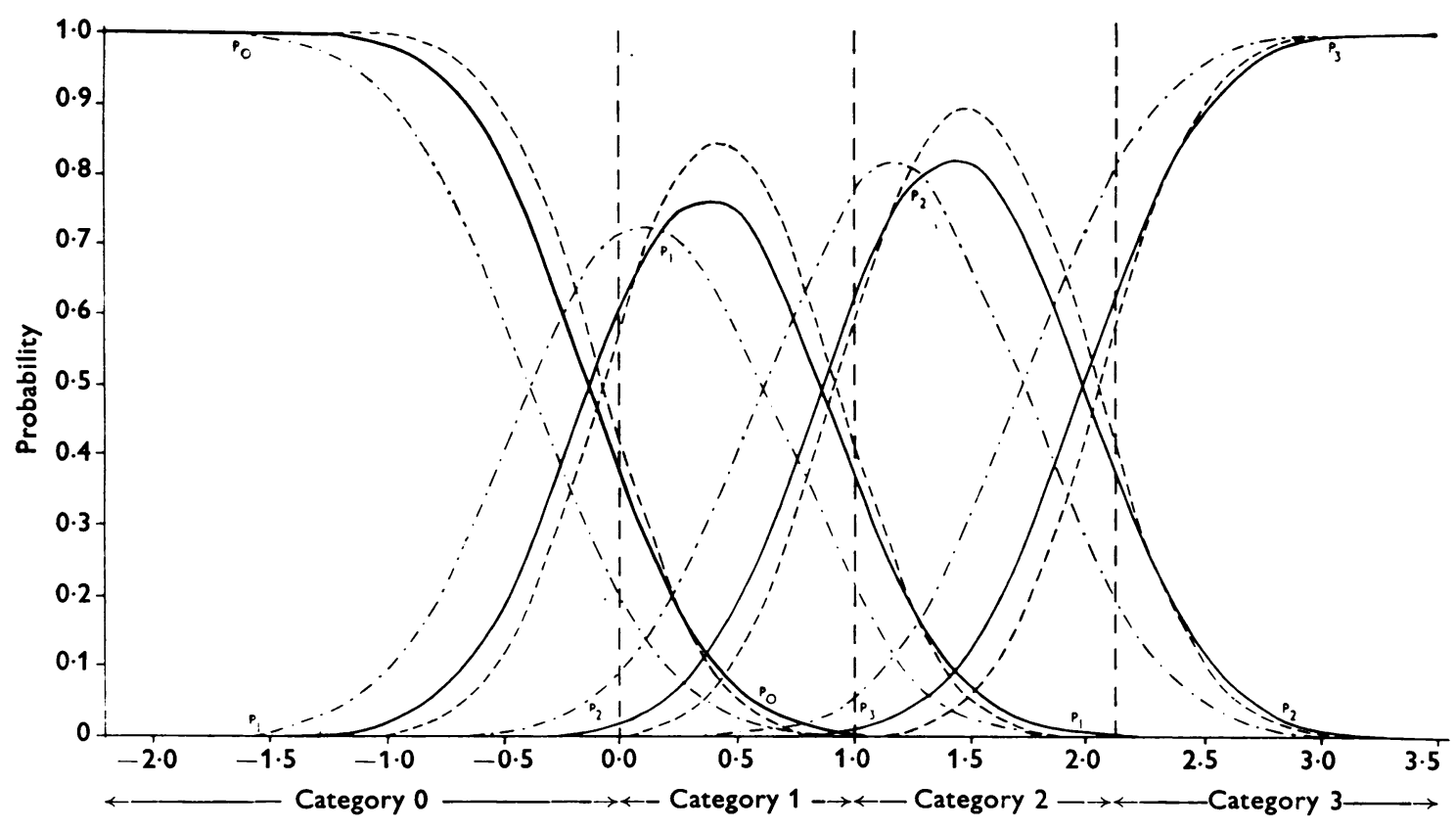

Abnormality

FIG. 2.-Colliery $\mathrm{C}_{11}$. Accuracy of the three sets of routine readings. First reading by Observer $A-$ -

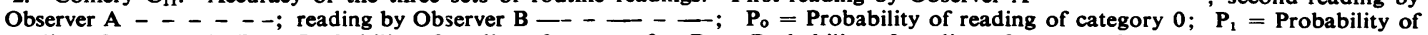
reading of category $1 ; \mathbf{P}_{2}=$ Probability of reading of category $2 ; \mathbf{P}_{3}=$ Probability of reading of category 3 .

of cases differences of two categories may be recorded. The probability curves for Observer A's second reading are very similar to those for his first reading. The peak values for the category 1 and category 2 curves are higher, reflecting the smaller variability of the second reading. The four curves relating to Observer B's readings are displaced to the left of those for Observer A. This is a reflection of the tendency for Observer B to read higher than Observer $A$, which makes the chance of his reading a film as category 0 smaller and increases the chance of his reading a film as category 1,2 or 3 .

It is interesting to note that the chance of reading a film with abnormality value of less than about $-2 \cdot 0$ as anything other than category 0 is negligible. Similarly, virtually all the films with abnormality values greater than 3.5 will be read as category 3 The chance of misclassification increases as the abnormality value approaches the category boundary, but even in the middle of categories 1 and 2 an appreciable proportion of the routine readings will be incorrect.

The Accuracy of the Sequences of Routine Readings.-It has been shown that the chance of assign- ing a film to any given category on a single routine reading depends both on the "true" abnormality value of the film and also on the reading error relating to the particular set of readings. Similarly, the chance of obtaining any given sequence of routine readings is a function of the true abnormality value and of the three sets of reading errors. The variation in the probabilities of obtaining the various sequences of routine readings is illustrated in Fig. 3, which also refers to colliery $C_{11}$.

The chance of obtaining the sequence $(0,0,0)$ decreases from unity for abnormality values below about -2.0 to 0.5 at $-0.5,0.03$ at 0 , and approaches zero beyond about $\mathbf{0 \cdot 2 5}$. It will be noted that a proportion of the films in the lower part of category 1 will be read as $(0,0,0)$. The curves for the sequences $(1,0,0),(0,1,0)$ and $(0,0,1)$ differ significantly both in position and shape, owing to the differences in standards on the three sets of readings. The maxima lie towards the upper limit of category 0 , although they occur at slightly different points on the scale. It should, however, be noted that the curves themselves cover a considerable range of abnormality values and that, for example, there is a chance of at least one in 20 of obtaining a reading 


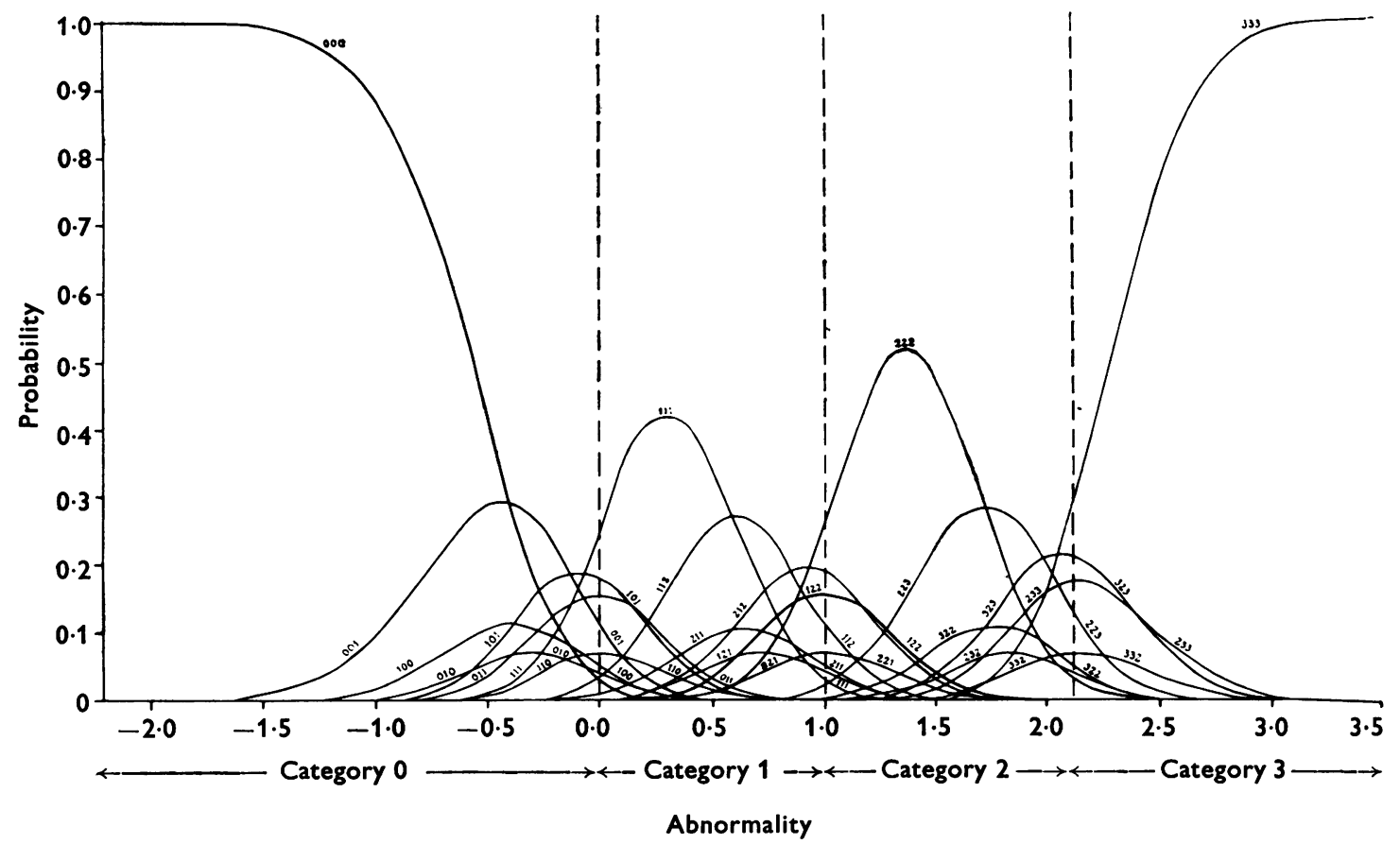

FIG. 3.-Colliery $C_{11}$. Accuracy of the various sequences of routine readings.

of $(0,0,1)$ between the points $-1 \cdot 2$ and 0.2 on the abnormality scale. The curves corresponding to the sequences $(0,1,1),(1,0,1)$ and $(1,1,0)$ follow a similar pattern, although they are displaced to the right. The curve corresponding to the sequence $(1,1,1)$ rises from zero for abnormality values below about -0.7 , to a maximum of just over 0.4 at 0.35 , and decreases to zero again beyond about $1 \cdot 3$. The curve corresponding to the sequence $(2,2,2)$ is similar to that for category 1 but attains a maximum of just over 0.5 .

Test of an "Averaging Procedure" to obtain the Definitive Classification of a Film.-The results given in Fig. 3 also serve as a warning against the indiscriminate application of any form of "averaging procedure" for the assessment of the definitive classification. The marked differences between the probability curves for sequences such as $(1,0,0)$, $(0,1,0)$ and $(0,0,1)$ emphasize the danger of regarding all sequences of category 0 twice and category 1 once as being representative of the same abnormality distribution. Even if the reading errors were constant on the three sets of routine readings (no bias, constant variance), in which case the curves for the sequences $(1,0,0),(0,1,0)$ and
$(0,0,1)$ would be coincident, the $(0,0,1)$ probability distribution would depend on the reading variance on the particular batch of films. On another batch of films, again with constant reading errors, the $(0,0,1)$ probability distribution would again depend on the reading variance, which might well be different from that on the first. Thus, even if the reading errors on the same batch of films were constant (which is most unlikely), readings of, say, $(0,0,1)$ on different batches of films would not necessarily be comparable. In summary, therefore, although a sequence of readings of the form $(0,0,1)$ does indicate on the average that the film is representative of a greater degree of abnormality than one read as $(0,0,0)$, it would be necessary to take account of the reading errors on each set of routine readings before any quantitative use could be made of this information. As to whether or not a film read as $(0,0,1)$ is actually to the left or right of the boundary between categories 0 and 1 , which is the major question both for progression studies and for statements of prevalence, reference to Fig. 3 shows that there is an appreciable chance of such a film belonging to either category. In this particular instance a great proportion of the films read as $(0,0,1)$ belong to category 0 , but it would obviously 
be wrong to assign all such films to this "average" reading.

It is perhaps worth emphasizing that the example dealt with in this section refers to the averaging of two readings by one observer and one by another. It will be quite obvious, however, that the argument applies equally well to any other method of averaging independent readings by two or more observers.

\section{Discussion and Conclusions}

The above illustrations show that the new model has a number of advantages. In the first place it can be applied to any particular film, provided the distribution of reading errors is known, as due account is taken of position on the abnormality scale within the category. The application of the model suggests that if a film is on the boundary between two categories it is equally likely to be read as either (if the reading bias is zero), and that as the true abnormality value moves away from the boundary the chance of misclassification decreases. In short, the new model gives a realistic representation of the actual mechanism of the reading process. From the point of view of simplicity of conception, once the initial difficulties over the choice of a scale of abnormality have been overcome, the description of the observer's performance in terms of only two parameters (his bias and variability) is obviously preferable to the multiplicity of $p_{i j}$ values implicit in the older model.

On the other hand the greater realism and simplicity of conception of the new model are obtained at the expense of a loss of universality of application, as the approach is confined to simple pneumoconiosis only. This is, of course, inevitable as the reading of simple pneumoconiosis and P.M.F. are completely separate processes. There is a further theoretical disadvantage, in that the model implies that the observer's bias is of the same "sign", i.e. either positive or negative, over the whole range of abnormality. For example, it is tacitly assumed that if he is "reading high" on the boundary between categories 0 and 1 he is also "reading high" on the boundaries between categories 1 and 2 and between categories 2 and 3. Now, it seems reasonable to suppose on general grounds that a definite bias on a particular set of readings is really due to the observer having drawn the borderlines between the categories at the wrong points on the scale of abnormality, and there is no theoretical reason why he should make the same kind of mistake on each of the three boundaries, although in the five examples considered this appears to have been the case. This difficulty is avoided in the $p_{i j}$ model, as each category boundary is considered separately.

The major practical disadvantage of the new model is the extent of the computations required to analyse the results in a satisfactory manner. The time taken to carry out the analysis is, in fact, comparable with the time taken for the readings. Analysis of our data shows that the "fit" of the new model is better, but not a great deal better, than that of the original model, and while the more realistic nature of the new model is an advantage, particularly from the point of view of the film reader, this cannot be measured in objective terms.

On balance, therefore, it appears that both models are of use and have particular applications, the one chosen depending upon the requirement of the research worker and the facilities available for computational work. Within the Pneumoconiosis Field Research the "improved" model has been used to a limited extent to explore some of the more interesting and obscure features of film reading and classification. On the other hand, its application to some of the more routine analyses of the results obtained at the selected collieries has not been considered justified, on the grounds of the extra computations involved. For example, in a companion paper, describing the analysis of all the film readings from the medical surveys conducted in the first six years of the research, the $p_{i j}$ model has been used as the basic method of approach, although, for the sake of simplicity of presentation, the results have been illustrated in the shape of orthodox "two-way" tables (Ashford, 1960).

This paper is published by permission of the National Coal Board, but the views expressed are not necessarily those of the Board. The work described is part of the Board's Pneumoconiosis Field Research.

Our thanks are due to Drs. S. Rae and P. J. Chapman, who read the films and provided constructive advice and criticism, and to Dr. J. Rogan and Mr. D. Hicks for their constant encouragement.

\section{REFERENCES}

Ashford, J. R. (1956). A Model for the Study of Observer Error in $X$-ray Reading. N.C.B. Report No. S.C. $366 / X R / 3$.

1957). The Accuracy of Classification by Joint Discussion. N.C.B. Report No. S.C. $479 /$ XR/4

(1959). A Problem of Subjective Classification in Industrial Medicine. Applied Statistics, VIII, 168.

Fay, J. W. J. (1957). Nature, 180, 309.

Fletcher, C. M., and Oldham, P. D. (1949). Brit. J. industr. Med., $6,168$.

International Labour Office (1953). Third International Conference of Experts on Pneumoconiosis, Sydney, 1950. Record of Proceedings, Geneva.

Wise, M. E. (1957). Private communication. 


\section{A P P E N D I X I}

Calculation of the Accuracy of the Definitive Classification

Method A.-The proportion of films of "true" category i, which are assigned to an incorrect "definitive" category, is given directly by $\left(1-p_{1 i}\right)$.

Method B.-When a film is classified under this procedure there are three possibilities: (a) the two routine readings will be consistent and correct, $(b)$ the two routine readings will be consistent but incorrect (in which case the film will be assigned to an incorrect "definitive" category), or (c) the two routine readings will be inconsistent (in which case the film will be classified by discussion). For a film of true category i the probabilities are given below:

\begin{tabular}{l|c|c}
\hline \multicolumn{1}{c|}{ Routine Readings } & Procedure & Probability \\
\hline Consistent and correct & No discussion & $\mathbf{p}_{11}{ }^{2}$ \\
Consistent but incorrect & No discussion & $\sum \mathbf{p}_{1 j^{2}}$ \\
Inconsistent & Discussion & $1-\underset{j}{\neq} \sum_{\mathbf{j}}{ }^{2}$ \\
\hline
\end{tabular}

Hence the probability of a film being assigned to an incorrect "definitive" category is

$$
\sum_{i \neq j} p_{i j}^{2}+0.2\left(1-\sum_{j} p_{i j}{ }^{2}\right) .
$$

Method C.-By a similar argument to that described above it can be shown that the probability of an incorrect "definitive" reading is $\sum_{i \neq j} p_{i j}{ }^{3}+0.2\left(1-\sum_{j} p_{i j}{ }^{3}\right)$.

Method D.-Under this procedure a film of "true" category 0 will be assigned to the correct "definitive" category without discussion for routine readings of $0,0,0 ; 1,0,0 ; 0,1,0$, and $0,0,1$, the film will be assigned to an incorrect "definitive" category without discussion for routine readings of $0,1,1 ; 1,0,1 ; 1,1,0 ; 1,1,1$, and other sequences differing by only one category which occur with a very low probability; the film will be discussed for routine readings of $0,1,2 ; 0,2,1 ; 1,0,2$; $1,2,0 ; 2,0,1 ; 2,1,0$, and other sequences differing by more than one category which occur with a very low probability.

The corresponding probabilities are respectively

$$
\mathrm{p}_{00}{ }^{3}+3 \mathrm{p}_{00}^{2} \mathrm{p}_{01} ; 3 \mathrm{p}_{01}{ }^{2} \mathrm{p}_{00}+\mathrm{p}_{01}{ }^{3}+\ldots .
$$
and; $6 \mathrm{p}_{00} \mathrm{p}_{\mathrm{ol}_{1}} \mathrm{p}_{\mathrm{og}}+$. .

The probability of classifying a film of "true" category 0 incorrectly is thus $\left[3 \mathrm{p}_{0_{1}}{ }^{2} \mathrm{p}_{0 o}+\mathrm{p}_{0_{1}}{ }^{3}+\ldots\right.$

$+0.2\left(6 p_{0 o} p_{01} p_{o_{2}}\right)+\ldots .$.

By a similar method it is possible to calculate the corresponding probabilities for the other true categories.

Method E.-The calculations are very similar to those for Method D, the main difference being the fact that films which differ by more than one category of simple pneumoconiosis are not discussed. 